\title{
Generalization of Sturm-Liouville Theory to a System of Ordinary Differential Equations with Dirac Type Spectrum
}

\author{
Chen Ning Yang \\ Institute for Theoretical Physics, State University of New York, Stony Brook, NY 11794, USA
}

Dedicated to Walter Thirring on his $60^{\text {th }}$ birthday

\begin{abstract}
The Sturm-Liouville theory is generalized to Dirac-equation-like systems of ordinary differential equations. It is shown how the comparison theorem and conversion to integral equations can be generalized.
\end{abstract}

\section{Introduction}

Some time ago [1] it was found necessary to generalize the Sturm-Liouville type of comparison theorems to coupled equations with a Dirac-like spectrum. The Sturm-Liouville theory deals with Schrödinger-like equations whose eigenvalues are bounded from below, i.e., equations which follow from a variational principle:

$$
\delta \int(\psi, H \psi) d x=0
$$

for normalized $\psi$ 's, where the integral is positive definite. Elegant comparison theorems in the Sturm-Liouville theory allow one to have powerful information on the number of eigenvalues, on the nodes of the wave functions and on the meaning of Levinson's theorem. Furthermore by converting the Sturm-Liouville problem to that of an integral operator with a symmetrical kernel, one has powerful control over properties of the eigenfunctions and eigenvalues.

In the present paper we shall show that all these can be generalized for a large class of Dirac-type equations in one variable, for which the eigenvalues extend to both $+\infty$ and $-\infty$. Use has already been made $[1,2]$ of these generalizations. The detail of the generalizations is published here for the first time.

The results of the present paper can be easily further generalized. E.g., one could deal with an Hermitian potential $V(x)$ rather than a real symmetrical one. One could generalize the matrix $\omega$ of (2.2), etc. No such generalizations are attempted in the present paper. 


\section{Differential Equation and Boundary Condition}

We consider the ordinary differential equation in matrix form

$$
\left[\omega \partial_{x}+V \psi\right]=E \psi, \quad(0 \leqq x \leqq a),
$$

where all quantities are real. $\psi$ is a $2 N \times 1$ column matrix, $V=V(x)$ is a real symmetrical $2 N \times 2 N$ matrix. We assume $V(x)$ to be continuous in the closed interval $(0, a)$, and

$$
\omega=\begin{array}{rr}
\mathbf{0} & -\mathbf{1} \\
\mathbf{1} & \mathbf{0}
\end{array}
$$

is an antisymmetrical $2 N \times 2 N$ matrix in which $\mathbf{1}=N \times N$ unit matrix.

We remark that a $4 \times 4$ Dirac eigenvalue equation is of the form (2.1) if we assume $\psi(x y z)$ to be independent of $y$ and $z$. Furthermore the radial equation discussed in [1] for a Dirac electron in a Dirac monopole field is also of the form (2.1).

We shall write

$$
\psi=\left(\begin{array}{l}
\xi \\
\eta
\end{array}\right),
$$

where $\xi$ and $\eta$ are $N \times 1$ column matrices. The boundary conditions at $x=0$ and $x=a$ are:

$$
\begin{array}{lll}
\xi=K_{0} \eta & \text { at } & x=0, \\
\xi=K_{a} \eta & \text { at } & x=a,
\end{array}
$$

where $K_{0}$ and $K_{a}$ are real symmetrical $N \times N$ matrices.

\section{Solution Set $\psi$ Satisfying Boundary Condition at $x=0$}

There are $N$ linearly independent solutions of (2.1) satisfying the boundary condition (2.4). These solutions can be successively defined by first, taking at

$$
x=0, \quad \eta=\left(\begin{array}{c}
1 \\
0 \\
0 \\
\vdots
\end{array}\right),\left(\begin{array}{c}
0 \\
1 \\
0 \\
\vdots
\end{array}\right),
$$

etc.; second, obtaining the corresponding $\xi$ 's at $x=0$ from (2.4); and third, integrating (2.1) from $x=0$ to $x=a$. We write these $N$ solutions, side by side, together as a $2 N \times N$ real matrix $\boldsymbol{\psi}$. Then

$$
\omega \partial_{x} \boldsymbol{\psi}+V \boldsymbol{\psi}=E \boldsymbol{\psi} .
$$

Write

$$
\psi=\left(\begin{array}{l}
\xi \\
\eta
\end{array}\right)
$$


Then

$$
\xi=K_{0}, \quad \boldsymbol{\eta}=\mathbf{1} \text { at } \quad x=0 .
$$

The most general solution of (2.1) satisfying the boundary condition (2.4) is thus a linear combination of the columns of $\psi$, i.e. $\psi \zeta$, where $\zeta$ is a $N \times 1$ column matrix.

Lemma 1. $\tilde{\boldsymbol{\psi}} \omega \boldsymbol{\psi}=0$ at all $x$.

Proof. At $x=0, \tilde{\boldsymbol{\psi}} \omega \boldsymbol{\psi}=-\tilde{\boldsymbol{\xi}} \boldsymbol{\eta}+\tilde{\boldsymbol{\eta}} \boldsymbol{\xi}=-\widetilde{K}_{0}+K_{0}=0$. Furthermore

$$
\frac{d}{d x}(\tilde{\boldsymbol{\psi}} \omega \boldsymbol{\psi})=\tilde{\boldsymbol{\psi}}(E-V) \boldsymbol{\psi}-\tilde{\boldsymbol{\psi}}(E-V) \boldsymbol{\psi}=0
$$

Lemma 2. $\tilde{\xi}_{\boldsymbol{\eta}}$ is symmetrical.

Proof. This lemma is an obvious consequence of Lemma 1.

Lemma 3. $\tilde{\boldsymbol{\psi}} \omega \frac{\partial \boldsymbol{\psi}}{\partial E}=\int_{0}^{x} \tilde{\boldsymbol{\psi}} \boldsymbol{\psi} d x>0$ for $x>0$.

Proof. Consider two neighboring $E^{\prime}$ s: $E_{1}$ and $E_{2}$.

$$
\begin{aligned}
& \omega \partial_{x} \boldsymbol{\psi}\left(E_{1}\right)=\left(E_{1}-V\right) \boldsymbol{\psi}\left(E_{1}\right), \\
& \omega \partial_{x} \boldsymbol{\psi}\left(E_{2}\right)=\left(E_{2}-V\right) \boldsymbol{\psi}\left(E_{2}\right) .
\end{aligned}
$$

Multiplying the transposed of (3.4) by $\psi\left(E_{2}\right)$ on the right and multiplying (3.5) by $\tilde{\psi}\left(E_{1}\right)$ on the left we obtain by subtraction

$$
-\left[\partial_{x} \tilde{\boldsymbol{\psi}}\left(E_{1}\right)\right] \omega \boldsymbol{\psi}\left(E_{2}\right)-\tilde{\boldsymbol{\psi}}\left(E_{1}\right) \omega \partial_{x} \boldsymbol{\psi}\left(E_{2}\right)=\left(E_{1}-E_{2}\right) \tilde{\boldsymbol{\psi}}\left(E_{1}\right) \boldsymbol{\psi}\left(E_{2}\right)
$$

Or

$$
\partial_{x}\left[\tilde{\boldsymbol{\psi}}\left(E_{1}\right) \omega \boldsymbol{\psi}\left(E_{2}\right)\right]=\left(E_{2}-E_{1}\right) \tilde{\boldsymbol{\psi}}\left(E_{1}\right) \boldsymbol{\psi}\left(E_{2}\right)
$$

Or

$$
\tilde{\boldsymbol{\psi}}\left(E_{1}\right) \omega \boldsymbol{\psi}\left(E_{2}\right)=\left(E_{2}-E_{1}\right) \int_{0}^{x} \tilde{\boldsymbol{\psi}}\left(E_{1}\right) \psi\left(E_{2}\right) d x .
$$

Differentiate both sides with respect to $E_{2}$ and put $E_{2}=E_{1}=E$. The result is Lemma 3.

\section{Properties of $\xi_{\eta^{-1}}$ and the Phase Angles $\theta_{i}$}

The matrices $\xi$ and $\boldsymbol{\eta}$ are functions of $E$ and $x$. They are what roughly correspond to $d_{x} \psi$ and $\psi$ for an ordinary Schrödinger equation integrated from $x=0$, with the boundary condition $d_{x} \psi=K, \psi=1$ at $x=0$. What roughly corresponds to the notion of a node is the value of $x$ where $\operatorname{det} \boldsymbol{\eta}=0$ (at some $E$ ). What roughly correspond to the logarithmic derivative $\psi^{-1} d_{x} \psi$ (which is so useful in elementary quantum mechanics) are the eigenvalues of the operator $\xi \eta^{-1}$. In this section we shall develop this last point in detail. 
Where $\operatorname{det} \boldsymbol{\eta} \neq 0, \boldsymbol{\xi} \boldsymbol{\eta}^{-1}=\tilde{\boldsymbol{\eta}}^{-1}(\tilde{\boldsymbol{\eta}} \xi) \boldsymbol{\eta}^{-1}$ is obviously real symmetrical, by Lemma 2 . It has $N$ real eigenvalues $\lambda_{1}, \lambda_{2} \ldots \lambda_{N}$. To study their variation with $E$, we first prove

Theorem 1. Where $\operatorname{det} \eta \neq 0$,

$$
\frac{\partial}{\partial E}\left(\boldsymbol{\xi} \boldsymbol{\eta}^{-1}\right)=\tilde{\boldsymbol{\eta}}^{-1}\left[\int_{0}^{x} \tilde{\boldsymbol{\psi}} \boldsymbol{\psi} d x\right] \boldsymbol{\eta}^{-1}
$$

Proof. Using (3.2) and Lemma 3 we have

$$
-\tilde{\xi} \frac{\partial \boldsymbol{\eta}}{\partial E}+\tilde{\boldsymbol{\eta}} \frac{\partial \xi}{\partial E}=\int_{0}^{x} \tilde{\boldsymbol{\psi}} \boldsymbol{\psi} d x
$$

By Lemma $2 \quad \tilde{\xi} \boldsymbol{\eta}=\tilde{\boldsymbol{\eta}} \xi$. Thus $\tilde{\xi}=\tilde{\boldsymbol{\eta}} \xi \boldsymbol{\eta}^{-1}$. Hence

$$
\left(-\tilde{\boldsymbol{\eta}} \xi \boldsymbol{\eta}^{-1}\right) \frac{\partial \boldsymbol{\eta}}{\partial E}+\tilde{\boldsymbol{\eta}} \frac{\partial \xi}{\partial E}=\int_{0}^{x} \tilde{\boldsymbol{\psi}} \boldsymbol{\psi} d x
$$

Or

$$
-\boldsymbol{\xi} \boldsymbol{\eta}^{-1} \frac{\partial \boldsymbol{\eta}}{\partial E} \boldsymbol{\eta}^{-1}+\frac{\partial \xi}{\partial E} \boldsymbol{\eta}^{-1}=\tilde{\boldsymbol{\eta}}^{-1}\left[\int_{0}^{x} \tilde{\boldsymbol{\psi}} \boldsymbol{\psi} d x\right] \boldsymbol{\eta}^{-1}
$$

Thus we have proved Theorem 1 .

Thus the eigenvalues of $\xi_{\eta^{-1}}: \lambda_{1}, \lambda_{2}, \ldots, \lambda_{N}$ are all monotonically increasing differentiable functions of $E$, provided $\operatorname{det} \boldsymbol{\eta} \neq 0$, and $x>0$. (This is true even if some of the $\lambda$ 's may be degenerate.)

We now investigate what happens at the point $E_{0}$ where $\operatorname{det} \boldsymbol{\eta}=0$. We first observe that $\lambda_{1}, \lambda_{2}, \ldots, \lambda_{N}$ are solutions of the polynomial equation

$$
\operatorname{det}(\xi-\lambda \boldsymbol{\eta})=0
$$

which has degree $N$ when $\operatorname{det} \boldsymbol{\eta} \neq 0$. At $E_{0}$ where $\operatorname{det} \boldsymbol{\eta}=0$, the equation misses the highest degree term whose coefficient is $\operatorname{det}(-\boldsymbol{\eta})$ which vanishes. It may miss the next few highest degree terms as well. Thus one or more of the solutions, say $\lambda_{1}$ and $\lambda_{2}$, may $\rightarrow \infty$ as $E \rightarrow E_{0}$. The rest of the solutions of (4.2) approach $\left(\lambda_{3}, \lambda_{4}, \ldots, \lambda_{N}\right)_{E_{0}}$. If $\operatorname{det} \xi \neq 0$ at $E_{0}$ we can consider $\boldsymbol{\eta} \xi^{-1}=\left(\boldsymbol{\xi} \boldsymbol{\eta}^{-1}\right)^{-1}$ and proceed similarly as above. However to take into consideration the possibility that $\operatorname{det} \xi$ may be $=0$, we proceed instead as follows. Let $A$ be a real number different from the values of $\lambda_{3}, \lambda_{4}, \ldots, \lambda_{N}$ at $E_{0}$. Then at $E_{0}$,

$$
\operatorname{det}(\xi-A \eta)=(\text { const })\left(A-\lambda_{3}\right)\left(A-\lambda_{4}\right) \ldots\left(A-\lambda_{N}\right) \neq 0 .
$$

We now consider the eigenvalues of $(\boldsymbol{\xi}-A \boldsymbol{\eta}) \boldsymbol{\eta}^{-1}=\boldsymbol{\xi} \boldsymbol{\eta}^{-1}-A$ in the neighborhood of $E_{0}$. They are $\lambda_{i}-A$, which approach $\infty, \infty, \lambda_{3}-A, \lambda_{4}-A, \ldots, \lambda_{N}-A$, none of which is zero. Thus we consider the inverse of $\xi_{\eta^{-1}}-A$, i.e.

$$
\boldsymbol{\eta}(\boldsymbol{\xi}-A \boldsymbol{\eta})^{-1},
$$

which exists in the neighborhood of $E_{0}$. The eigenvalues of (4.3) are $\left(\lambda_{i}-A\right)^{-1}$. Now we can prove, in the same way that we proved Theorem 1,

$$
\frac{\partial}{\partial E}\left[\boldsymbol{\eta}(\boldsymbol{\xi}-A \boldsymbol{\eta})^{-1}\right]=-(\boldsymbol{\xi}-A \boldsymbol{\eta})^{-1}\left[\int_{0}^{x} \tilde{\boldsymbol{\psi}} \boldsymbol{\psi} d x\right](\boldsymbol{\xi}-A \boldsymbol{\eta})^{-1}
$$


Thus near $E=E_{0}$ where $\operatorname{det} \boldsymbol{\eta}=0,\left(\lambda_{i}-A\right)^{-1}$ are all monotonically decreasing differentiable functions of $E$, provided $x>0$. If follows from this trivially that $\lambda_{3}, \lambda_{4}, \ldots, \lambda_{N}$ are all differentiable at $E=E_{0}$. So are $\frac{1}{\lambda_{1}}$ and $\frac{1}{\lambda_{2}}$.

The results above can be summarized as the following theorem.

Theorem 2. The eigenvalues of $\boldsymbol{\xi}_{\boldsymbol{\eta}^{-1}}$ can be written as $\lambda_{i}=\tan \theta_{i}, i=1 \rightarrow N$, where $\frac{\partial \theta_{i}}{\partial E}>0$ for all $i$, if $x>0$.

We remark here that at $\theta_{i}=\frac{\pi}{2}(\bmod \pi), \lambda_{i}= \pm \infty$, but $\theta_{i}$ remains a continuous and differentiable function of $E$. Obviously the function

$$
\theta_{i}(E, x) \text {, }
$$

can be changed by adding to it, for a fixed $x$, an integral multiple of $\pi$. To eliminate this freedom we require $\theta_{i}(E, x)$ to be continuous with respect to $x$, and take

$$
\begin{aligned}
\theta_{i}(E, 0)= & \tan ^{-1}\left[\mathrm{i}^{\text {th }} \text { eigenvalue of } K_{0}\right], \\
& -\frac{\pi}{2} \leqq \theta_{i}(E, 0)<\frac{\pi}{2} .
\end{aligned}
$$

With this convention, $\theta_{i}(E, x)$ is continuous in both $E$ and $x$, and is further differentiable with respect to $E$, with $\partial \theta / \partial E>0$ for all $x>0$. We shall call the $\theta$ 's the phase angles. Later on we shall show that for $x>0$, as $E \rightarrow+\infty$, all $\theta_{i} \rightarrow+\infty$, and as $E \rightarrow-\infty$, all $\theta_{i} \rightarrow-\infty$.

It is instructive to think of $\theta_{i}(\bmod \pi)$ in the form of a ring as illustrated in Fig. 1. As $E$ increases, each $\theta_{i}$ winds around the cylinder in a left-handed spiral. Can spirals $i$ and $j(i \neq j)$ cross? They can. To construct an example of this one takes two independent problems each defined by (2.1) and (2.4), with $\psi$ 's of sizes $2 N_{1} \times 1$ and $2 N_{2} \times 1$, and puts them together to make a single problem with a $\psi$ of size $2\left(N_{1}+N_{2}\right) \times 1$. The two sets of independent spirals can of course be made to cross. But as soon as coupling between these two independent problems are introduced, the crossing disappears in the usual way as illustrated in Fig. 2. In general, therefore, the different spirals do not cross.

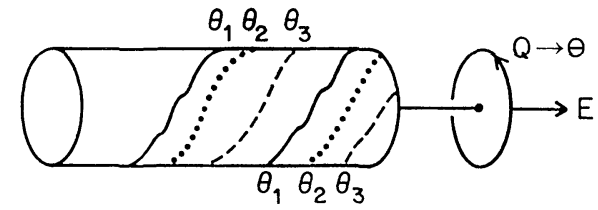

Fig. 1

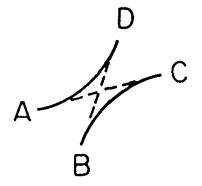

Fig. 2

Fig. 1. $\theta_{1}, \theta_{2}, \theta_{3}$, vs. $E$ as left handed spirals. Notice that $d \theta_{i} / d E$ is always positive

Fig. 2. Noncrossing of $\theta_{i}$ and $\theta_{j}$. Without coupling one could have $\theta_{i}$ following $A C$ and $\theta_{j}$ following $B D$, which cross each other. A small coupling would in general switch the $\theta_{i}, \theta_{j}$ trajectories to the solid curves $A D$ and $B C$ which avoid crossing 
As an example we consider the case where $V=0$. Equation (3.1) becomes

$$
-\partial_{x} \boldsymbol{\eta}=E \xi, \quad \partial_{x} \xi=E \boldsymbol{\eta} .
$$

The solution of (4.7) satisfying (3.3) is

$$
\begin{gathered}
\xi=(\cos E x) K_{0}+(\sin E x) \mathbf{1}, \\
\boldsymbol{\eta}=-(\sin E x) K_{0}+(\cos E x) \mathbf{1} .
\end{gathered}
$$

Thus

$$
\xi \boldsymbol{\eta}^{-1}=\frac{(\tan E x) \mathbf{1}+K_{0}}{1-(\tan E x) K_{0}} .
$$

If the diagonal form of $K_{0}$ is

$$
K_{0}=A\left(\begin{array}{ccc}
\tan \alpha_{1} & & 0 \\
& \tan \alpha_{2} & \\
0 & & \ddots
\end{array}\right) \tilde{A}
$$

then

$$
\xi \boldsymbol{\eta}^{-1}=A\left(\begin{array}{ccc}
\tan \left(E x+\alpha_{1}\right) & \\
& \tan \left(E x+\alpha_{2}\right) & \\
0 & \ddots
\end{array}\right) \widetilde{A}
$$

Thus

$$
\theta_{i}=E x+\alpha_{1}
$$

\section{Eigenvalues $E_{\ell}$ and Eigenfunctions $\phi_{\ell}$}

In Sects. 3 and 4 we have not imposed the boundary condition (2.5) at $x=a$. Imposing this boundary condition leads to the eigenvalues of the problem, a development that we shall pursue in this section. In order to emphasize the symmetry of the two boundary conditions we adopt a procedure already familiar in the usual [4] Sturm-Liouville theory: Consider a solution set $\psi_{1}$ of the equation

$$
\omega \partial_{x} \boldsymbol{\psi}_{1}+V \boldsymbol{\psi}_{1}=E \boldsymbol{\psi}_{1}
$$

just like Eq. (3.1), but with $\boldsymbol{\psi}_{1}$ satisfying the boundary condition (2.5) at $x=a$, rather than (2.4) at $x=0$. I.e.

$$
\boldsymbol{\psi}_{1}=\left(\begin{array}{c}
K_{a} \\
1
\end{array}\right) \text { at } x=a .
$$

We integrate (5.1) from $x=a$ backwards towards $x=0$, and define

$$
W(x)=\tilde{\boldsymbol{\psi}}_{1} \omega \psi
$$


It is trivially clear that one has the following lemmas:

\section{Lemma 4.}

$$
\frac{d}{d x} W(x)=0
$$

\section{Lemma 5.}

$$
W=\left.\left(-K_{a} \eta+\xi\right)\right|_{x=a} .
$$

In Sect. 3 we have seen that the most general solution of (3.1) satisfying the boundary condition at $x=0$ is

$$
\left(\begin{array}{l}
\xi \\
\eta
\end{array}\right) \zeta .
$$

For this solution to also satisfy the boundary condition (2.5) at $x=a$ means

$$
(\xi)_{x=a} \zeta=K_{a}(\boldsymbol{\eta})_{x=a} \zeta
$$

By (5.5) this implies $W \zeta=0$. Thus one has

Lemma 6. A necessary and sufficient condition that there is a nonvanishing solution of (2.1), satisfying both boundary conditions (2.4) and (2.5), is $\operatorname{det} W=0$. Such a solution is called an eigenfunction and the corresponding value of $E$ an eigenvalue. For a given eigenvalue the number of linearly independent eigenfunctions is equal to the number of linearly independent '’s satisfying $W \zeta=0$.

The number of independent eigenfunctions for a given eigenvalue is called the latter's degeneracy. An eigenvalue is nondegenerate if its degeneracy is unity.

Lemma 7. If $\psi_{\ell}$ and $\psi_{m}$ are eigenfunctions belonging to two different eigenvalues $E_{\ell}$ and $E_{m}$, then

$$
\int_{0}^{a} \tilde{\psi}_{\ell} \psi_{m} d x=0
$$

and we say that $\psi_{\ell}$ and $\psi_{m}$ are orthogonal.

The proof is entirely similar to the usual one and will be omitted.

Lemma 8. All eigenvalues are real.

Proof. If eigenvalue $E_{\ell}$ is not real and $\psi_{\ell}$ is an eigenfunction belonging to $E_{\ell}$, then $E_{\ell}^{*}=E_{m}$ is also an eigenvalue and $\psi_{\ell}^{*}$ one of its eigenfunctions. Equation (5.6) then states

$$
\int_{0}^{a} \tilde{\psi}_{\ell} \psi_{\ell}^{*}=0
$$

which implies $\psi_{\ell}=0$, a contradiction.

For a degenerate eigenvalue $E_{\ell}$, we can choose the eigenfunctions at $E_{\ell}$ to be mutually orthogonal. We thus have 
Theorem 3. The problem (2.1) with boundary conditions (2.4) and (2.5) have eigenvalues $E_{\ell}$ which are all real. The corresponding eigenfunctions $\phi_{\ell}$ can be and will be chosen to be mutually orthogonal.

Furthermore we shall always normalize the eigenfunctions $\phi_{\ell}$.

\section{Density of Eigenvalues}

For the case $V=0, \xi \eta^{-1}$ is periodic in $E$ with period $\pi / a$, according to (4.11). The eigenvalues are values of $E$, where $\operatorname{det}\left(\xi_{\eta} \eta^{-1}-K_{a}\right)=0$. Thus the eigenvalues form $N$ series, in each of which the eigenvalues are equally spaced with spacing $=\pi / a$.

If $V(x)=B \mathbf{1}$, where $B$ is a numerical constant and 1 the $2 N \times 2 N$ unit matrix, then in Eq. (2.1) the term $V \psi$ can be moved to the right-hand side of the equation, resulting in changing $E$ to $E-B$. Thus the eigenvalues again form $N$ series, in each of which the eigenvalues are equally spaced with spacing $=\pi / a$.

For the general case $V(x) \neq 0$, consider for each $x$ the lowest eigenvalue $V_{\text {l.b. }}(x)$ of the real symmetrical matrix $V(x)$. Let $V_{1.1 . b}$ be the lower bound of $V_{\text {l.b. }}(x)$ in the interval $0 \leqq x \leqq a$. We write

$$
\begin{gathered}
V_{0}(x)=V_{\text {l.1.b. }}, \\
V_{1}(x)=V(x)-V_{1}(x) .
\end{gathered}
$$

Obviously $V_{1}(x)$ is a positive matrix for all $x$ in $0 \leqq x \leqq a$, and its largest eigenvalue is bounded from above by a number, to be denoted by $K$.

Now consider the problem

$$
\left[\omega \partial_{x}+\left(V_{0}+\lambda V_{1}\right)\right] \psi=E \psi
$$

with the boundary conditions (2.4) and (2.5). We shall denote the eigenvalues of the problem by

$$
E_{\ell}(\lambda)
$$

$$
\begin{gathered}
E_{\ell}(1)=\text { eigenvalues for problem with } V=V(x), \\
E_{\ell}(0)=\text { eigenvalues for problem with } V=V_{0} .
\end{gathered}
$$

According to what we had proved above, $E_{\ell}(0)$ form $N$ series of equally spaced eigenvalues. We shall so define $E_{\ell}(\lambda)$ that they are continuous in $\lambda$. According to perturbation theory,

$$
\frac{d E_{\ell}(\lambda)}{d \lambda}=\left\langle\left|V_{1}\right|\right\rangle
$$

Thus

$$
K \geqq \frac{d E_{\ell}(\lambda)}{d \lambda} \geqq 0
$$

Integrate with respect to $\lambda$ from $\lambda=0$ to $\lambda=1$. We obtain

$$
K \geqq E_{\ell}(1)-E_{\ell}(0) \geqq 0 \text {. }
$$


Consider any interval $(\alpha, \beta)$ of energy $\alpha<E \leqq \beta$ : Let $M(\alpha, \beta)$ be the number of eigenvalues in this interval for the problem $V=V(x)$, and $M_{0}(\alpha, \beta)$ that for the problem $V=V_{0}$. Equation (6.7) implies that

$$
M_{0}(\alpha-K ; \beta) \geqq M(\alpha, \beta) \geqq M_{0}(\alpha, \beta-K) .
$$

Now

$$
\begin{aligned}
& M_{0}(\alpha, \beta-K)=M_{0}(\alpha, \beta)-M_{0}(\beta-K, \beta), \\
& M_{0}(\alpha-K, \beta)=M_{0}(\alpha, \beta)+M_{0}(\alpha-K, \alpha),
\end{aligned}
$$

and $M_{0}(\beta-K, \beta)$ is $\leqq$ a number $\gamma$ which is independent of $\beta$, because the eigenvalues $E_{\ell}(0)$ form a periodic pattern. Similarly

$$
M_{0}(\alpha-K, \alpha) \leqq \gamma
$$

Thus

$$
M_{0}(\alpha, \beta)+\gamma \geqq M(\alpha, \beta) \geqq M_{0}(\alpha, \beta)-\gamma .
$$

Now in each interval of length $\pi / a$ there are exactly $N$ states for $V_{0}$. I.e.

$$
M_{0}\left(\alpha, \alpha+\pi a^{-1}\right)=N \text {. }
$$

Thus

$$
\left|M_{0}(\alpha, \beta)-(\beta-\alpha) \pi^{-1} N a\right| \leqq N
$$

Combining (6.8) and (6.9) we obtain

\section{Theorem 4.}

$$
\left|M(\alpha, \beta)-(\beta-\alpha) \pi^{-1} N a\right| \leqq N+\gamma .
$$

Notice that $N+\gamma$ is independent of $\alpha$ and $\beta$. Equation (6.10) implies that the average density of eigenvalues in a large interval is $\approx N a / \pi$.

\section{Conversion to Integral Equation with Symmetrical Kernel}

The usual Sturm-Liouville theory has been converted into a linear integral equation with a symmetrical kernel by Hilbert, Dixon and others (see [4]). We shall show in the present section that this development can be generalized to the problem studied in the present paper, i.e. Eqs. (2.1), (2.4), and (2.5) which have a Dirac spectrum.

Let $\varepsilon$ be any real number which is not an eigenvalue of (2.1), (2.4), and (2.5). We write, for $E=\varepsilon$, the solutions $\psi$ of (3.2), $\psi_{1}$ of (5.1) and the $W$ matrix of (5.3) as

$$
\boldsymbol{\psi}(x, \varepsilon), \boldsymbol{\psi}_{1}(x, \varepsilon) \text {, and } W(x, \varepsilon) .
$$

$W(x, \varepsilon)$ is actually independent of $x$. Notice that $\operatorname{det} W \neq 0$. We now define a $(2 N \times 2 N)$ matrix-integral operator $\Omega$ such that when it operates on a $2 N \times 1$ column $f(y)$, a new column $\Phi(x)$ results:

$$
\Phi(x)=\int_{0}^{a}\langle x|\Omega| y\rangle f(g) d y .
$$


or

$$
\Phi(x)=\boldsymbol{\psi}_{1}(x, \varepsilon) \tilde{W}^{-1}(x, \varepsilon) \int_{0}^{x} \tilde{\boldsymbol{\psi}}(y, \varepsilon) f(y) d y+\boldsymbol{\psi}(x, \varepsilon) W^{-1}(x, \varepsilon) \int_{x}^{a} \tilde{\boldsymbol{\psi}}_{1}(y, \varepsilon) f(y) d y .
$$

We assume here that $f(y)$ is piecewise continuous. The explicit expression of $\Omega$ is a matrix:

for $x \geqq y$,

$$
\langle i x|\Omega| j y\rangle=\left\langle i\left|\boldsymbol{\psi}_{1}(x, \varepsilon)\right| b\right\rangle\left\langle c\left|W^{-1}(x, \varepsilon)\right| b\right\rangle\langle j|\psi(y, \varepsilon)| c\rangle
$$

for $x<y$,

$$
\langle i x|\Omega| j y\rangle=\langle i|\psi(x, \varepsilon)| b\rangle\left\langle b\left|W^{-1}(x, \varepsilon)\right| c\right\rangle\left\langle j\left|\psi_{1}(y, \varepsilon)\right| c\right\rangle .
$$

Notice that indices $i$ and $j$ run from 1 to $2 N$ while indices $b$ and $c$ run from 1 to $N$. Notice that $\Omega$ is symmetrical:

$$
\langle j y|\Omega| i x\rangle=\langle i x|\Omega| j y\rangle .
$$

We shall write (7.1) symbolically as $\Phi=\Omega f$.

Lemma 9. If $f$ is piecewise continuous, then (a) Eq. (7.1) defines a continuous $\Phi(x)$ which is piecewise differentiable and satisfies

$$
(\varepsilon-H) \Phi=f,
$$

where

$$
H=\omega \partial_{x}+V
$$

(b) $\Phi(x)$ satisfies the boundary conditions (2.4) and (2.5) for $\psi$ at $x=0$ and $x=a$.

(c) $\Phi(x)$ is the only solution of (7.4) satisfying the boundary conditions mentioned above.

Proof. (a) By straight forward differentiation, we obtain from (7.1)

$$
(\varepsilon-H) \Phi=\omega\left[\boldsymbol{\psi} W^{-1} \tilde{\boldsymbol{\psi}}_{1}-\boldsymbol{\psi}_{1} \tilde{W}^{-1} \tilde{\boldsymbol{\psi}}\right] f=\omega\left(\boldsymbol{\psi}_{1} \boldsymbol{\psi}\right)\left(\overline{W^{-1}} \mid \frac{-\tilde{W}^{-1}}{}\right)\left(\frac{\tilde{\boldsymbol{\psi}}_{1}}{\tilde{\boldsymbol{\psi}}}\right) f .(7.6)
$$

Now write the three matrices inbetween $\omega$ and $f$ on the right-hand side of (7.6) as $B_{1}, B_{2}$, and $\widetilde{B}_{1}$. By Lemma 1 and definition (5.3),

$$
\widetilde{B}_{1} \omega B_{1}=\frac{W}{-\tilde{W}} \mid \frac{W}{2}=B_{2}^{-1}
$$

Thus

$$
\widetilde{B}_{1} \omega B_{1} B_{2}=1
$$

I.e.

$$
\omega B_{1} B_{2} \widetilde{B}_{1}=1,
$$

which leads to (7.4). 
(b) At $x=0, \Phi=\boldsymbol{\psi}(0, \varepsilon)$ [column matrix], by (7.1). Equation (3.3) then shows that $\Phi(x)$ at $x=0$ satisfies the correct boundary condition. Similarly one can prove that $\Phi(x)$ also satisfies the boundary condition at $x=a$.

(c) If there were another solution of (7.4) satisfying the same boundary conditions, denote it by $\Phi_{1}$. Then $(\varepsilon-H)\left(\Phi-\Phi_{1}\right)=0$. Since $\varepsilon$ is not an eigenvalue, this implies that $\Phi=\Phi_{1}$.

This completes the proof of Lemma 9.

Thus the operation (7.1) symbolically written as

$$
\Phi=\Omega f
$$

has the inverse operation

$$
(\varepsilon-H) \Phi=f,
$$

if one starts with a piecewise continuous $f$.

Lemma 10. Every eigenfunction [5] $\phi_{\ell}$ of $H$ with eigenvalue $E_{\ell}$ is also an eigenfunction of $\Omega$, with eigenvalue $\left(\varepsilon-E_{\ell}\right)^{-1}$, so that

$$
\Omega \phi_{\ell}=\left(\varepsilon-E_{\ell}\right)^{-1} \phi_{\ell} .
$$

Proof.

$$
(\varepsilon-H) \phi_{\ell}=\left(\varepsilon-E_{\ell}\right) \varphi_{\ell}
$$

We shall write both sides as $f$ :

$$
\begin{aligned}
(\varepsilon-H) \varphi_{\ell} & =f, \\
\left(\varepsilon-E_{\ell}\right) \varphi_{\ell} & =f .
\end{aligned}
$$

Equation (7.10) shows that $f$ is continuous. Define, as in (7.1)

$$
\Phi=\Omega f .
$$

Then $\Phi$ satisfies the boundary conditions and

$$
f=(\varepsilon-H) \Phi .
$$

Eliminating $f$ from (7.12) and (7.9), we obtain

$$
(\varepsilon-\mathrm{H}) \varphi_{\ell}=(\varepsilon-H) \Phi .
$$

Since $\varphi_{\ell}-\Phi$ satisfy the correct boundary conditions and $\varepsilon$ is not an eigenvalue of $H$, we have

$$
\varphi_{\ell}=\Phi \text {. }
$$

Operate with $\Omega$ on (7.10) and use (7.11) and (7.13);

$$
\left(\varepsilon-E_{\ell}\right) \Omega \phi_{\ell}=\Phi=\phi_{\ell}
$$

This proves the lemma.

Lemma 11. $\Omega$ does not have zero as an eigenvalue. Every eigenfunction $g_{\ell}$ of $\Omega$ with eigenvalue $\mu_{\ell}$ is also an eigenfunction [5] of $H$ with eigenvalue

$$
\varepsilon-\mu_{\ell}^{-1} \text {. }
$$


Proof. The eigenfunctions of $\Omega$ are [6] piecewise continuous. Now $\Omega g_{\ell}=\mu_{\ell} g_{\ell}$. Lemma 9 states that $(\varepsilon-H)\left(\Omega g_{\ell}\right)=g_{\ell}$. Thus

$$
\mu_{\ell}(\varepsilon-H) g_{\ell}=g_{\ell} .
$$

It follows from this that $\mu_{\ell} \neq 0$. Hence $g_{\ell}=\mu_{\ell}^{-1}\left(\Omega g_{\ell}\right)$. Lemma 9 then shows that $g_{\ell}$ is continuous, piecewise differentiable and satisfies the boundary conditions at $x=0$ and $x=a$.

Now (7.14) implies

$$
H g_{\ell}=\left(\varepsilon-\mu_{\ell}^{-1}\right) g_{\ell}
$$

which proves the lemma.

Lemmas 10 and 11 identify the eigenfunctions of $H$ with those of the integral operator $\Omega$. We can then use the powerful results [6] on expansion theorems for integral operators for the differential operator $H$.

\section{References}

1. Yang, C.N.: Monopoles in quantum field theory. Craigie, Goddard, Nahm (eds.). Singapore: World Scientific 1982, p. 237

2. Ma, Z.Q.: Levinson's theorem for Dirac particles moving in a background magnetic monopole field, and Levinson's theorem for Dirac particles with a long-range potential. Phys. Rev. D 32 , 2203 , and 2213 (1985)

3. For comparison theorems see e.g. Reid, W.T.: Applied mathematical sciences, Vol. 31. Berlin, Heidelberg, New York: Springer 1980

4. See e.g. Titmarsh, E.C.: Eigenfunction expansions. Part I. Oxford: Oxford University Press

5. We mean by this that $H \phi_{\ell}=E_{\ell} \phi_{\ell}$ and that $\phi_{\ell}$ satisfies the boundary conditions at $x=0$ and $x=a$

6. Courant, R., Hilbert, D.: Methods of mathematical physics, Vol. I. New York: Interscience 1953

Communicated by E. H. Lieb

Received February 11, 1987 